\title{
Quantifying Heterogeneity in Tumors: Proposing a New Method Utilizing Convolutional Neuronal Networks
}

\author{
Georg PROKOP ${ }^{\mathrm{a}, 1}$, Michael ÖRTL $^{\mathrm{b}}$, Marina FOTTELER ${ }^{\mathrm{b}}$, Peter SCHÜFFLER $^{\mathrm{c}}$, \\ Johannes SCHOBEL ${ }^{\mathrm{b}}$, Walter SWOBODA ${ }^{\mathrm{b}}$, Jürgen SCHLEGEL ${ }^{\mathrm{a}}$ and Friederike \\ LIESCHE-STARNECKER ${ }^{\mathrm{a}}$ \\ ${ }^{a}$ Department of Neuropathology, Institute of Pathology, Technical University of \\ Munich, GERMANY \\ ${ }^{\mathrm{b}}$ DigiHealth Institute, Neu-Ulm University of Applied Sciences, GERMANY \\ ${ }^{c}$ Department of Computational Pathology, Institute of Pathology, Technical University \\ of Munich, GERMANY
}

\begin{abstract}
Heterogeneity is a hallmark of glioblastoma (GBM), the most common malignant brain tumor, and a key reason for the poor survival rate of patients. However, establishing a clinically applicable, cost-efficient tool to measure and quantify heterogeneity is challenging. We present a novel method in an ongoing study utilizing two convolutional neuronal networks (CNN). After digitizing tumor samples, the first CNN delimitates GBM from normal tissue, the second quantifies heterogeneity within the tumor. Since neuronal networks can detect and interpret underlying and hidden information within images and have the ability to incorporate different information sets (i.e. clinical data and mutational status), this approach might venture towards a next level of integrated diagnosis. It may be applicable to other tumors as well and lead to a more precision-based medicine.
\end{abstract}

Keywords. Tumor heterogeneity, Glioblastoma, Convolutional Neuronal Network, Neuropathology, Digital Pathology

\section{Introduction}

Cancer heterogeneity is ubiquitous: For many years, human cancers are known to be heterogenous in their gene expression and morphological appearance [1,2]. Since tumor heterogeneity is believed to play a key role in acquiring therapy resistance and thus leading to a poor prognosis in patients, there is great interest in it [3, 4]. Most studies use large-scale mutational characterization to qualify and quantify cancer heterogeneity. While these methods are useful to describe and discover intra- and intertumoral differences, they lack extensive accessibility. Thus, there is a need for easily available and clinically applicable methods to quantify tumor heterogeneity, ideally aided by computational technologies [5].

\footnotetext{
${ }^{1}$ Corresponding Author, Georg Prokop, Department of Neuropathology, School of Medicine, Institute of Pathology, Technical University Munich, Trogerstraße 18, 81675 München, Germany; E-mail: georg.prokop@tum.de.
} 
Artificial intelligence is a promising and powerful tool: Among these computational technologies, artificial intelligence (AI) is one of the most promising emerging approaches. Certain types of AI, especially convolutional neuronal networks $(\mathrm{CNN})$, have successfully been applied in different areas of medical image analysis. Among others, they perform at least equal to a dermatologist in detecting skin-cancer in images [6,7] and predict patient outcome from colorectal cancer tissue [8]. It is also possible to train neuronal networks to integrate different types of data, such as images and genomic biomarkers to generate more accurate predictions of survival [9]. With this powerful tool at hand, one can tackle other complex problems and questions like the automatic quantification of heterogeneity [10].

Heterogeneity is a hallmark of glioblastoma: As its original name "glioblastoma multiforme" suggests, glioblastoma (GBM) is known to be inter- and intratumorally heterogenous [11]. It is also infamous for its poor median survival rate of 15 months [12]. Currently, GBM is diagnosed according to the WHO classification based on histological and molecular criteria creating a so called integrated diagnosis [13]. Since heterogeneity is considered a hallmark of GBM and several studies describing it have been published, it is an ideal specimen to test our novel approach for automated quantification of heterogeneity $[11,14]$.

In the ongoing study described in this manuscript, we combine histological images with mutational features that account for tumor heterogeneity, thus resulting in a next level of integrated diagnostics. By using easily available hematoxylin and eosin (HE) slides as input (standard diagnostic slides), we ensure our trained networks to be applicable in various settings, helping on the road towards precision-based medicine. This manuscript focuses on the main approach and methodology in our current research.

\section{Method}

\subsection{Overall Layout}

We quantify heterogeneity by using CNNs to detect underlying and hidden information in digital HE slides. We train two separate networks using supervised machine learning. The first network differentiates between tumor tissue and surrounding brain tissue in $\mathrm{HE}$ slides. The second network is set up to measure heterogeneity in the tumor regions in the HE slides defined by the first CNN. As network architectures, we test both pre-trained networks like the Google Inception V3 and an untrained network architecture. To avoid overfitting, we augment our data by rotating and flipping the input images and use dropout regularization. The ground truth for training are manually labelled HE slides and heterogeneity data derived from immunohistochemical (IHC) staining of seven proteins. However, the final pipeline will only use HE slides to both detect tumor lesions and quantify their heterogeneity (see Figure 1). This study was approved by the local ethics committee (vote no. 164/19 S-SR, chair Prof. Dr. G. Schmidt).

\subsection{Tumor detection}

Acquisition and pre-processing of image data: As a training data set, we use 58 GBM tissue samples stained with HE. 54 tumor-free reference slides from brain autopsies 


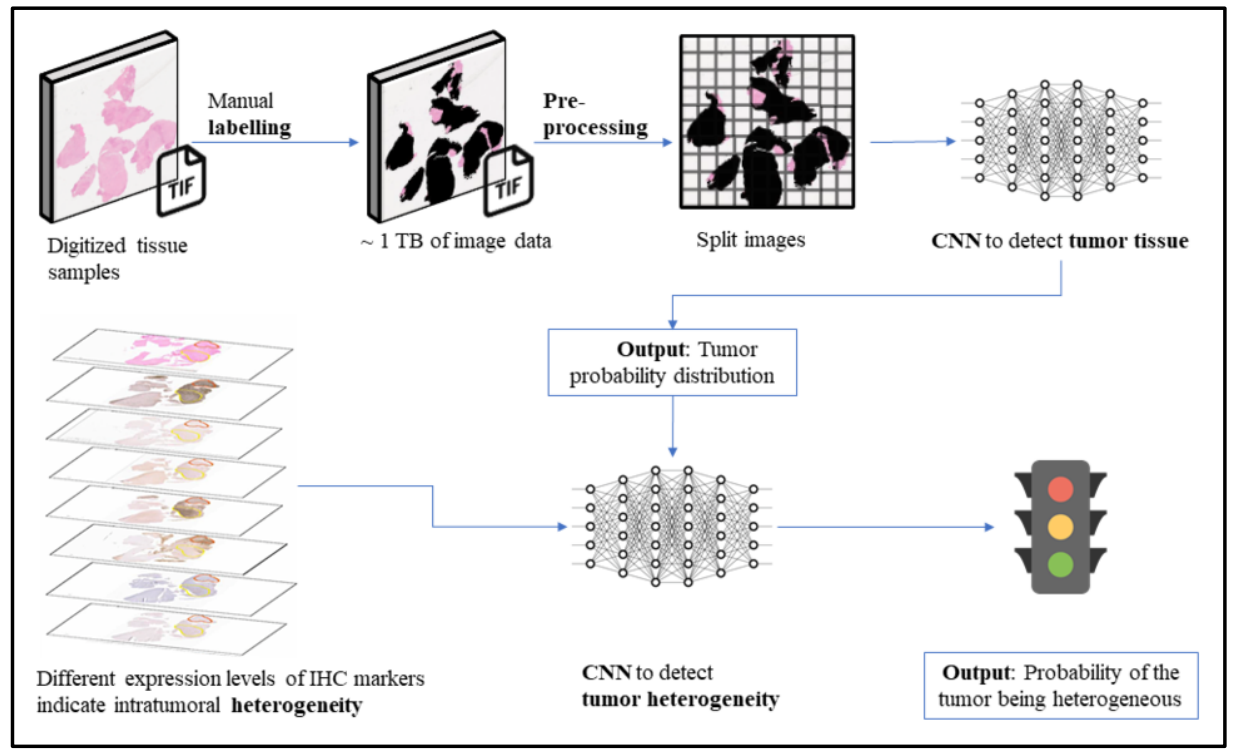

Figure 1. Training the Tumor Heterogeneity Measurement Pipeline: Together with control samples from

brain autopsies, the tumor images are used to train a $\mathrm{CNN}$ to delimitate healthy and tumor tissue and secondly to measure tumor heterogeneity based on a immunohistochemically derived classification system.

served as controls. After digitizing the slides, the core regions of the tumorous lesions were manually labelled and separated from the surrounding brain tissue. Altogether this resulted in over $1 \mathrm{~TB}$ of image data (available as TIF files). As pre-processing steps, the images were split into smaller images patches creating approx. 278.900 training samples per slide.

Processing and analyzing the images: The aforementioned smaller patches are analyzed by a CNN in a sliding window approach. Starting with a 300 by 300 pixel frame, the network determines whether the central core region of that patch contains tumor tissue by using all the information in the whole frame. Thus, all the information in the frame is condensed onto its core. Next, the frame is shifted to the side by the central core region. Thereby, the whole image is analyzed creating a probability distribution of the core regions containing background, tumor or healthy tissue.

\subsection{Heterogeneity measurement}

We determined the overall heterogeneity of the tumor sample using a previously described method based on the different expression levels of seven IHC markers [14]. Training the second $\mathrm{CNN}$ incorporates the heterogeneity data, provided as a classification of the tumors in 'homogenous', 'intermediate' or 'heterogenous', and the previously derived tumor distribution to determine the probability of the HE slide being derived from a heterogenous or homogenous tumor sample. Thus, this probability serves as a surrogate parameter of heterogeneity itself.

\subsection{Validation}

To validate our pipeline, we chose various approaches: Firstly, we randomly split our training data into two patient groups. The first group, about $80 \%$ of the total patients, is 
the actual training data set, the second, about $20 \%$, serves as a validation data set. Here, the network can prove its capabilities. Secondly, the detected tumor tissue is shown in the original image using a color code, indicating different levels of tumor probability. In the future this may help pathologists to identify tumor areas more easily. Additionally, we will validate our networks in publicly available image sets like the TCGA data set or the Ivy Atlas. Finally, we will correlate our findings to patient survival. The source code will be made publicly available.

\section{Conclusion}

This article describes the method of an ongoing project to quantify heterogeneity in tumors and GBM in particular. As heterogeneity, especially of GBM, is of utmost importance for therapy decisions and thus patient-survival, this project will contribute to a better treatment in neurooncology. By incorporating patient data into a multimodal learning algorithm, we want to advance precision-based medicine and provide a next level of integrated diagnosis.

\section{References}

[1] Heppner GH. Tumor Heterogeneity. Cancer Research. 1984;44(6):2259-65.

[2] Andor N, Graham TA, Jansen M, Xia LC, Aktipis CA, Petritsch C, et al. Pan-cancer analysis of the extent and consequences of intratumor heterogeneity. Nat Med. 2016;22(1):105-13.

[3] Dagogo-Jack I, Shaw AT. Tumour heterogeneity and resistance to cancer therapies. Nat Rev Clin Oncol. 2018;15(2):81-94.

[4] Lee J-K, Wang J, Sa JK, Ladewig E, Lee H-O, Lee I-H, et al. Spatiotemporal genomic architecture informs precision oncology in glioblastoma. Nature Genetics. 2017;49(4):594-9.

[5] McGranahan N, Swanton C. Clonal Heterogeneity and Tumor Evolution: Past, Present, and the Future. Cell. 2017;168(4):613-28.

[6] Esteva A, Kuprel B, Novoa RA, Ko J, Swetter SM, Blau HM, et al. Dermatologist-level classification of skin cancer with deep neural networks. Nature. 2017;542(7639):115-8.

[7] Brinker TJ, Hekler A, Enk AH, Klode J, Hauschild A, Berking C, et al. Deep learning outperformed 136 of 157 dermatologists in a head-to-head dermoscopic melanoma image classification task. Eur J Cancer. 2019;113:47-54.

[8] Bychkov D, Linder N, Turkki R, Nordling S, Kovanen PE, Verrill C, et al. Deep learning based tissue analysis predicts outcome in colorectal cancer. Sci Rep. 2018;8(1):3395.

[9] Mobadersany P, Yousefi S, Amgad M, Gutman DA, Barnholtz-Sloan JS, Velázquez Vega JE, et al. Predicting cancer outcomes from histology and genomics using convolutional networks. Proceedings of the National Academy of Sciences. 2018;115(13):E2970-E9.

[10] Zhong Q, Ruschoff JH, Guo T, Gabrani M, Schuffler PJ, Rechsteiner M, et al. Image-based computational quantification and visualization of genetic alterations and tumour heterogeneity. Sci Rep. 2016;6:24146.

[11] Verhaak RG, Hoadley KA, Purdom E, Wang V, Qi Y, Wilkerson MD, et al. Integrated genomic analysis identifies clinically relevant subtypes of glioblastoma characterized by abnormalities in PDGFRA, IDH1, EGFR, and NF1. Cancer Cell. 2010;17(1):98-110.

[12] Stupp R, Mason WP, van den Bent MJ, Weller M, Fisher B, Taphoorn MJ, et al. Radiotherapy plus concomitant and adjuvant temozolomide for glioblastoma. N Engl J Med. 2005;352(10):987-96.

[13] Louis DN, Perry A, Wesseling P, Brat DJ, Cree IA, Figarella-Branger D, et al. The 2021 WHO Classification of Tumors of the Central Nervous System: a summary. Neuro Oncol. 2021;23(8):1231-51.

[14] Liesche-Starnecker F, Mayer K, Kofler F, Baur S, Schmidt-Graf F, Kempter J, et al. Immunohistochemically Characterized Intratumoral Heterogeneity Is a Prognostic Marker in Human Glioblastoma. Cancers (Basel). 2020;12(10). 